\title{
Stardust in the Laboratory
}

\author{
Ernst Zinner ${ }^{\mathrm{A}}$ \\ A Laboratory for Space Sciences and the Physics Department, Washington University, \\ St. Louis, MO 63130, USA. Email: ekz@wustl.edu
}

Received 2007 October 6, accepted 2007 November 26

\begin{abstract}
Primitive meteorites and interplanetary dust particles contain small grains that originated in stellar outflows and supernova explosions. These $\mu \mathrm{m}$ - and sub- $\mu \mathrm{m}$-sized presolar grains can be isolated and studied for their isotopic compositions in the laboratory. They are recognised as stardust by their isotopic compositions, which are completely different from those of the Solar System. They provide new information on stellar evolution, nucleosynthesis, mixing processes in asymptotic giant branch (AGB) stars and supernovae, and Galactic chemical evolution. Red giants, AGB stars, Type II supernovae and possibly novae have been identified as stellar sources of the grains. Of the eight nuclear processes proposed by Burbidge et al. (1957), signatures of all except the $r$-process can be found in presolar dust grains.
\end{abstract}

Keywords: nuclear reactions, nucleosynthesis, abundances — stars: AGB and post-AGB — stars: supernovae: general — dust, extinction

\section{Introduction}

Fifty years ago, following the proposal by Hoyle (1946) that the elements from carbon on up to the actinides are produced by nuclear reactions in stars, the classic papers by Burbidge et al. (1957, henceforth $\left.\mathrm{B}^{2} \mathrm{FH}\right)$ and Cameron (1957) established stellar nucleosynthesis as a scientific discipline (e.g. Wallerstein et al. 1997). These authors proposed eight different nuclear processes occurring in different types of stars to produce the chemical elements heavier than helium in nature. The newly synthesised elements are injected by stellar winds or as supernova (SN) ejecta into the interstellar medium where they form the starting material for new generations of stars. The Solar System is believed to have formed from a complicated mixture of material from many stellar sources, integrating over a long Galactic history. The work of $\mathrm{B}^{2} \mathrm{FH}$ and Cameron was actually stimulated by regularities in the abundance of the nuclides in Solar System material as obtained from the study of meteorites (Suess \& Urey 1956). Another important stimulus was provided by the observation of the unstable element Tc in the spectra of S-stars (Merrill 1952). Although the Solar System composition represents only a grand average of many stellar sources, the Solar System (= cosmic) abundances of the elements and isotopes (Cameron 1973; Anders \& Grevesse 1989; Grevesse, Noels \& Sauval 1996; Lodders 2003; Asplund, Grevesse \& Sauval 2005) became an important reference for astronomers and cosmochemists. They remain a touchstone for testing models of stellar nucleosynthesis (e.g. Käppeler, Beer \& Wisshak 1989; Timmes, Woosley \& Weaver 1995).

In the last twenty years, a new source of information on isotopic abundances in stars has become available in the form of stardust preserved in primitive meteorites, interplanetary dust particles, micrometeorites and comets. Small dust grains that condensed in the expanding atmospheres of red giant branch and asymptotic giant branch (AGB) stars and in the ejecta of supernovae survived travel in the interstellar medium and were included in the molecular cloud from which our Solar System formed. Some of these grains survived in meteorites from which they can be extracted and analysed by various techniques in the laboratory. Their study provides information on stellar nucleosynthesis, stellar evolution, Galactic chemical evolution, physical properties in stellar atmospheres, mixing of SN ejecta and conditions in the Solar nebula and the parent stars of meteorites in which these grains are found. This new field of astronomy has grown to an extent that a detailed treatment is not possible in the framework of the present paper. The reader is invited to consult some recent reviews (Zinner 1998; Hoppe \& Zinner 2000; Nittler 2003; Clayton \& Nittler 2004; Hoppe 2004; Lodders \& Amari 2005; Lugaro 2005; Zinner 2007). Here I want to concentrate on a limited aspect, namely to what extent the eight nuclear processes proposed by $\mathrm{B}^{2} \mathrm{FH}$ and Cameron are reflected in the isotopic compositions of stardust grains in meteorites and what constraints on stellar nucleosynthetic processes can be obtained from them.

\section{Types of Stardust and Isotopic Analysis}

The first types of stardust grains (also called presolar grains) discovered were the carbonaceous phases diamond (Lewis et al. 1987), silicon carbide (SiC) (Bernatowicz et al. 1987; Tang \& Anders 1988) and graphite (Amari et al. 1990). The reason is that these phases carry isotopically anomalous 'exotic' noble gas components. By tracking the noble gas signatures in chemically treated meteorite 
Table 1. Presolar grain types

\begin{tabular}{lccll}
\hline Grain type & $\begin{array}{c}\text { Abundance } \\
\text { ppm }\end{array}$ & $\begin{array}{c}\text { Size } \\
\mu \mathrm{m}\end{array}$ & Stellar sources & $\begin{array}{l}\text { Nucleosynthetic processes }^{\mathrm{b}} \\
\text { exhibited by grains }^{\mathrm{a}}\end{array}$ \\
\hline Nanodiamonds & 1400 & 0.002 & $\mathrm{SNe}$ & $\mathrm{r}, \mathrm{p}$ \\
Silicates in IDPs & $\sim 400$ & $\leq 1$ & RGB and AGB & Core H \\
Silicates in meteorites & 180 & $\leq 0.5$ & RGB and AGB & Core H, CBP \\
Oxides & 110 & $0.15-2$ & RGB, AGB, SNe & Core H, CBP, HBB, Shell H, He, s \\
Mainstream SiC & 14 & $0.3-20$ & AGB & Core H, Shell H, Shell He, s \\
SiC type A + B & 0.25 & $0.5-5$ & J stars? & Shell He and H \\
SiC type X & 0.15 & $0.3-5$ & SNe & $\mathrm{H}, \mathrm{He}, \mathrm{O}, \mathrm{e}, \mathrm{s}, \mathrm{n}$-burst \\
Graphite & $\sim 10$ & $1-20$ & $\mathrm{SNe}, \mathrm{AGB}$ & $\mathrm{H}, \mathrm{He}, \mathrm{O}, \mathrm{e}, \mathrm{s}, \mathrm{n}$-burst; Core H and He \\
Nova grains (SiC, gr.) & 0.001 & $\sim 1$ & Novae & Ex H \\
Si nitride & 0.002 & $\leq 1$ & SNe & $\mathrm{He}, \mathrm{O}$ \\
TiC & $\sim 0.001$ & $0.01-0.5$ & SNe, AGB & $\mathrm{He}, \mathrm{O}, \mathrm{e}$ \\
\hline
\end{tabular}

${ }^{a}$ Abundances (in parts per million) vary with meteorite type. Shown here are the maximum values.

bIn low-to-intermediate-mass stars: 'Core H' is core $\mathrm{H}$ burning followed by first (and second) dredge-up; 'Shell H' is shell $\mathrm{H}$ burning during the RG and AGB phase; 'Shell He' is He burning during thermal pulses of the AGB phase followed by third dredge-up; 'CBP' is cool bottom processing; 'HBB' is hot bottom burning; ' $\mathrm{s}$ ' is $s$-process, neutron capture at low neutron density, followed by third dredge-up. In supernovae: $\mathrm{H}, \mathrm{He}, \mathrm{O}: \mathrm{H}, \mathrm{He}$ and $\mathrm{O}$ burning in different stellar zones in the massive star before explosion; ' $\mathrm{s}$ ' is the $s$-process taking place in several zones; ' $\mathrm{e}$ ' is the equilibrium process, leading to the Fe-Ni core; 'n-burst' is neutron capture at intermediate neutron density; ' $r$ ' is neutron capture at high neutron density; ' $\mathrm{p}$ ' is the $p$-process, photo disintegration and proton capture. In novae: 'Ex H' is Explosive H burning.

samples, Ed Anders and his colleagues at the University of Chicago succeeded in isolating the carrier grains (see Anders \& Zinner 1993).

Further isotopic measurements of these grains confirmed that they are bona fide stardust that originated from stellar sources because of their extremely anomalous isotopic compositions. Although the abundance of these carbonaceous presolar grains in meteorites is low (see Table 1), almost pure samples are obtained by chemical and physical separation (Amari, Lewis \& Anders 1994). This provides enough material for 'bulk' isotopic analysis, the analysis of collections of large numbers of grains by gas mass spectrometry of C and N (Russell, Arden \& Pillinger 1996; Russell et al. 1997) and the noble gases (Lewis, Amari \& Anders 1994; Amari, Lewis \& Anders 1995), by thermal ionization mass spectrometry of Sr, Ba and other heavy elements (e.g. Hoppe \& Ott 1997), and inductively coupled plasma mass spectrometry (Yin, Lee \& Ott 2006). While bulk analysis only provides compositions averaged over many grains, it allows the measurement of trace elements such as the noble gases and heavy elements that cannot be obtained otherwise.

However, since stardust grains come from different stellar sources, information on individual stars is obtained from the analysis of single grains. For isotopic analysis of single grains, the technique of choice is secondary ion mass spectrometry (SIMS) in the ion microprobe. Single-grain isotopic analysis has also been possible by laser ablation and resonant ionization mass spectrometry (RIMS). Because of its high sensitivity, RIMS analysis has been successfully applied to the heavy elements zirconium, molybdenum, ruthenium and barium (see, e.g. Savina et al. 2003). The ion microprobe has been instrumental in the discovery of other types of stardust grains such as corundum and spinel (Hutcheon et al. 1994; Nittler et al. 1994), silicon nitride (Nittler et al. 1995) and silicates (Messenger et al. 2003; Nguyen \& Zinner 2004). Additional types of presolar grains such as Ti-, Zr- and Morich carbides, Fe carbide, kamacite (Fe-Ni) and elemental Fe were discovered as tiny subgrains during transmission electron microscope (TEM) studies of graphite and $\mathrm{SiC}$ (Bernatowicz et al. 1996; Croat et al. 2003).

Table 1 lists presolar grain types, their abundances in meteorites and interplanetary dust particles (IDPs), sizes, stellar sources and the nucleosynthetic signatures carried by the grains.

Nanodiamonds are too small to be analysed as single grains. Their presolar nature rests on the fact that they carry anomalous $\mathrm{Xe}$ and $\mathrm{Te}$. However, their $\mathrm{C}$ isotopic ratio is normal (solar) and it cannot be excluded that only a fraction of diamonds have a stellar origin.

Figure 1 shows electron microscope images of different presolar grain types. Average sizes of presolar grains are less than $1 \mu \mathrm{m}$ but grains in excess of $20 \mu \mathrm{m}$ have been also found. Figures 1a-d show unusually large grains. Such large grains allow the isotopic analysis of many elements. The difficulty of locating presolar grains increases steeply from $\mathrm{SiC}$ and graphite to silicate grains. Essentially all $\mathrm{SiC}$ grains and at least half of graphite grains are isotopically anomalous and thus of stellar origin. Because in the Solar System O $>$ C, these phases do not form there. However, O-rich minerals form in the Solar System and represent a background to the few O-rich stardust grains. Only $\sim 1-2 \%$ of corundum and spinel grains are stardust. Although silicates are the most abundant presolar grains after nanodiamonds (Table 1), only one of 5000 silicate grains is of stellar origin because of the overwhelming presence of isotopically normal silicates of Solar System 

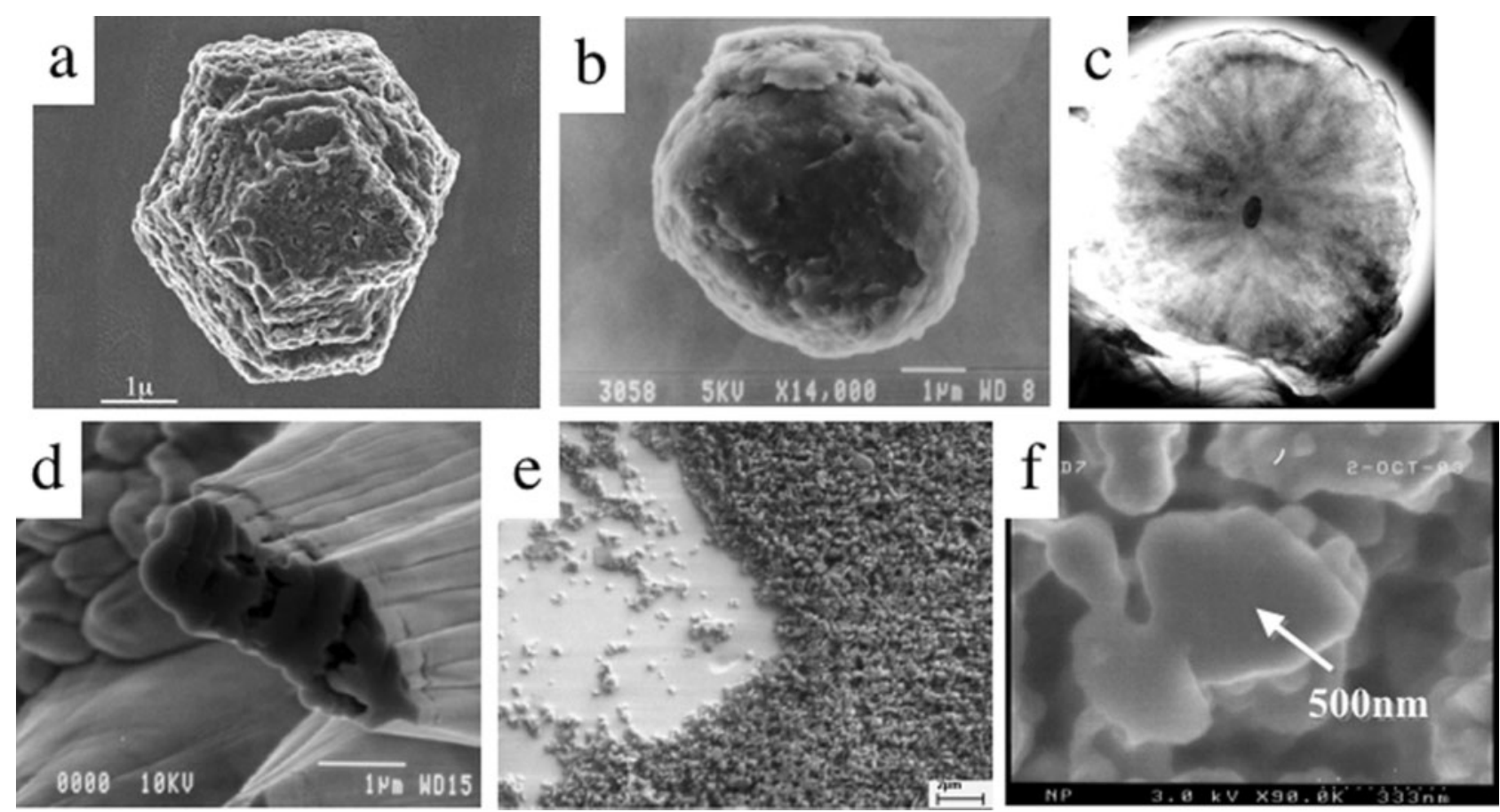

Figure 1 Scanning electron micrographs of presolar dust grains. (a) Silicon carbide, (b) Graphite, (c) TEM image of graphite slice with interior TiC grain, (d) Aluminum oxide, (e) Spinel (only $\sim 2 \%$ of the grains in this image are presolar), (f) Silicate.

origin. The location of presolar silicates requires the isotopic analysis of many thousands of grains. This has been made possible by raster imaging of the $\mathrm{O}$ isotopes in the NanoSIMS (Nguyen, Zinner \& Lewis 2003; Nguyen \& Zinner 2004) or by direct imaging with the Cameca IMS 1270 equipped with a SCAPS (stacked CMOS-type active pixel sensor) device (Yurimoto, Nagashima \& Kunihiro 2003; Nagashima, Krot \& Yurimoto 2004).

Let us compare the different sources of information on isotopic abundances in stars: the Solar System abundances, astronomical measurements of stars (e.g. Lambert 1991; Yong, Lambert \& Ivans 2003) and presolar grains from meteorites. The Solar System abundances include all the elements and their stable isotopes, but the Solar System is a mix of many different stellar sources. Astronomical isotopic measurements have the advantage that they are made on known stars. However, such measurements are limited to cool stars, are made for only a limited number of elements and usually have low precision. Stardust grains provide relatively high precision data of correlated elements and give isotopic information on stars that cannot be observed astronomically (e.g. supernovae). However, the stellar sources of the grains are unknown and have to be inferred from the isotopic compositions of the grains. This has led to a close interplay between laboratory analysis of grains and theoretical predictions from nucleosynthetic models of different types of stellar sources (Woosley \& Weaver 1995; Gallino, Busso \& Lugaro 1997b; Gallino et al. 1998; Rauscher et al. 2002; José et al. 2004).

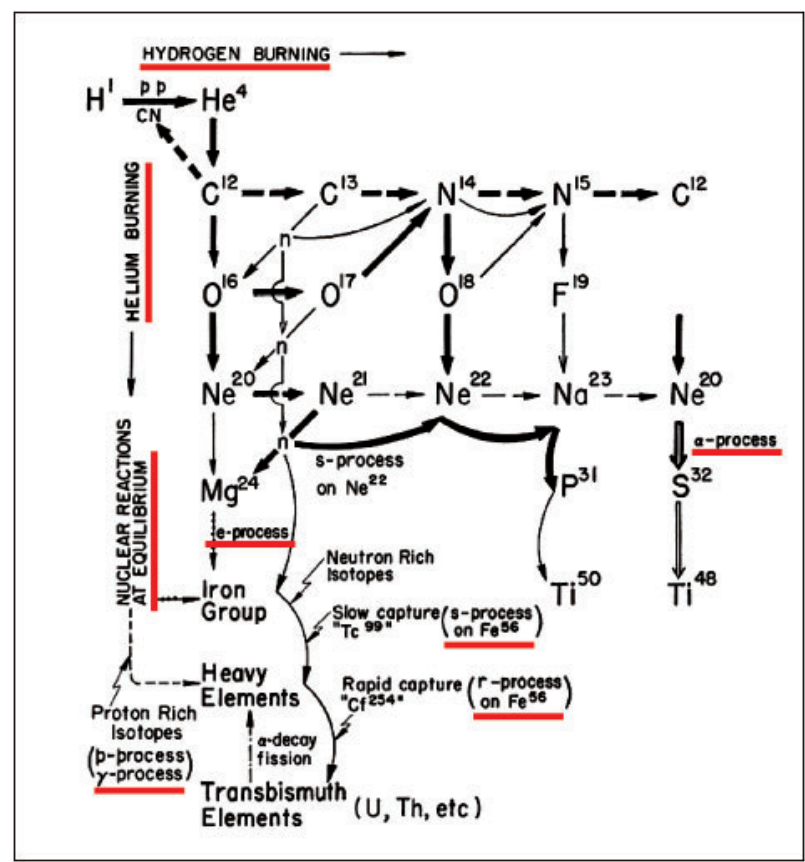

Figure 2 Schematic diagram of the nuclear processes for the synthesis of the elements in stars identified by $\mathrm{B}^{2} \mathrm{FH}$. This is taken from their figure I,2.

\section{Nucleosynthetic Processes Reflected in the Isotopic Compositions of Presolar Grains}

Figure 2 shows the schematic diagram of the stellar nucleosynthetic processes from the $\mathrm{B}^{2} \mathrm{FH}$ paper. The 


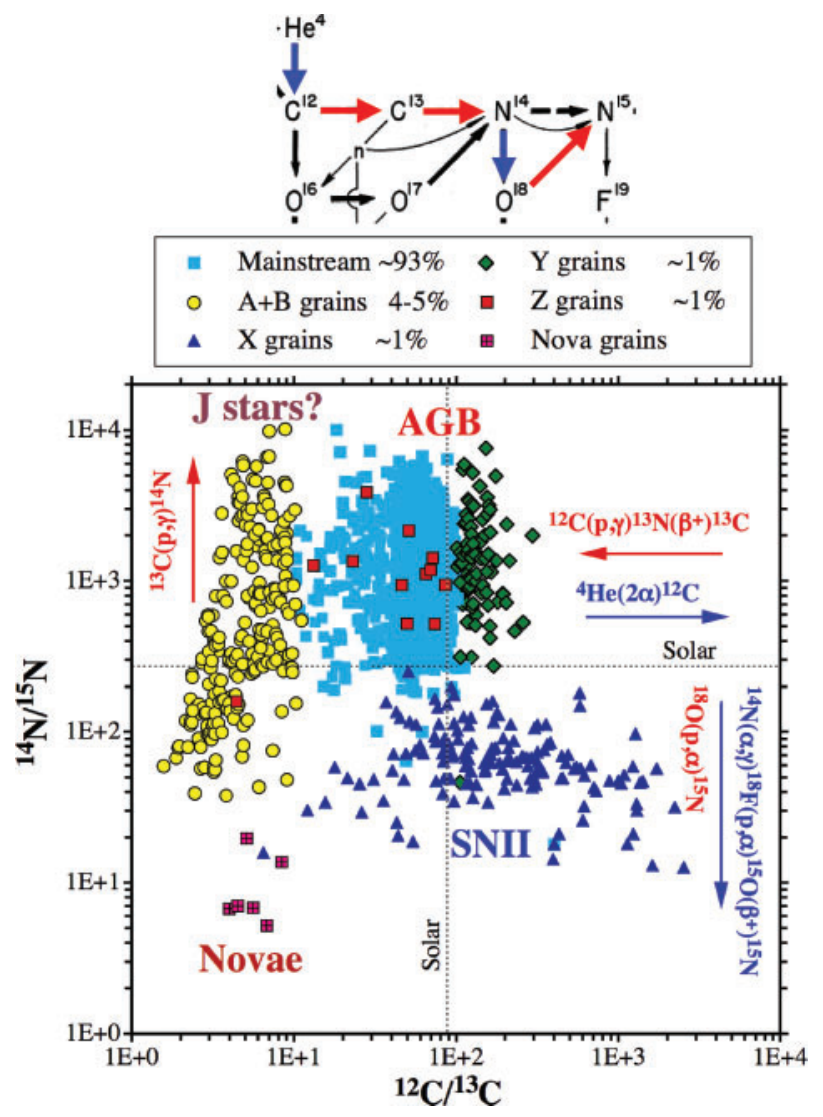

Figure 3 Nitrogen $v s . \mathrm{C}$ isotopic ratio plot of single presolar $\mathrm{SiC}$ grains. Different classes of presolar $\mathrm{SiC}$ grains, $\mathrm{p}$ - and $\alpha$-capture reactions, and the inferred stellar sources of the grains are indicated. The top of the picture shows the $\mathrm{p}$ - and $\alpha$-capture reactions from $\mathrm{B}^{2} \mathrm{FH}$.

nucleosynthetic processes identified by these authors are underlined in red.

In the following I will show that the signatures of most of these processes can be found in presolar grains. In some of the plots of isotopic compositions presented, the relevant portion of the diagram of Figure 2 is inserted.

\subsection{Hydrogen and Helium Burning}

Signs of $\mathrm{H}$ and He burning are prominently displayed in the range of isotopic compositions measured in presolar $\mathrm{SiC}$, graphite and oxide grains. Figure 3 shows the $\mathrm{C}$ and $\mathrm{N}$ isotopic ratios measured in presolar $\mathrm{SiC}$ grains.

Note that the isotopic ratios are shown on a log-log plot. Both the $\mathrm{C}$ and $\mathrm{N}$ isotopic ratios cover a range of almost four orders of magnitude. Indicated are the nuclear reactions that shift the isotopic compositions in different directions on the plot. Proton capture on ${ }^{12} \mathrm{C}$ and subsequent $\beta^{+}$decay produces ${ }^{13} \mathrm{C}$ and therefore lowers the ${ }^{12} \mathrm{C} /{ }^{13} \mathrm{C}$ ratio. The triple- $\alpha$ reaction produces ${ }^{12} \mathrm{C}$ and increases the ${ }^{12} \mathrm{C} /{ }^{13} \mathrm{C}$ ratio. Proton capture produces both ${ }^{14} \mathrm{~N}$ and ${ }^{15} \mathrm{~N}$, depending whether if happens on ${ }^{13} \mathrm{C}$ or ${ }^{18} \mathrm{O}$, whereas $\alpha$ capture followed by $\mathrm{p}$ capture and $\beta^{+}$decay produces ${ }^{15} \mathrm{~N}$. The nuclear reactions are possible production pathways but are not sufficient to explain the ratios observed in the grains. For this it is necessary to know in which stellar sources und under which conditions the reactions occurred. Close interaction with nuclear astrophysicists who work on stellar models is therefore indispensable. What makes identification of stellar sources easier is that different types of presolar grains have characteristic isotopic ratios in several elements (see below).

There is convincing evidence, mainly from the isotopic compositions of heavy elements (e.g. Gallino et al. 1997a; Hoppe \& Ott 1997; Lugaro et al. 2003; Savina et al. 2004) that mainstream, $\mathrm{Y}$ and $\mathrm{Z}$ grains originate from low-mass AGB stars with a range of metallicities. The $\mathrm{C}$ and $\mathrm{N}$ isotopic ratios in the parent stars is first determined by $\mathrm{H}$ burning in the CNO cycle during the hydrostatic phase of the stars followed by first (and second) dredge-up (El Eid 1994; Gallino et al. 1994), resulting in low ${ }^{12} \mathrm{C} /{ }^{13} \mathrm{C}$ and high ${ }^{14} \mathrm{~N} /{ }^{15} \mathrm{~N}$ ratios. During the thermally pulsing AGB phase, ${ }^{12} \mathrm{C}$ produced in the He-burning shell is mixed into the envelope during recurrent third dredge-up episodes, increasing the ${ }^{12} \mathrm{C} /{ }^{13} \mathrm{C}$ and the $\mathrm{C} / \mathrm{O}$ ratio (Zinner et al. 2006). When $\mathrm{C}>\mathrm{O}$ the star becomes a carbon star and $\mathrm{SiC}$ grain can condense in its expanding atmosphere. However, models of low-metallicity AGB stars predict much higher ${ }^{12} \mathrm{C} /{ }^{13} \mathrm{C}$ ratios than those observed in $\mathrm{Z}$ grains whose $\mathrm{Si}$ and Ti isotopes indicate an origin in such stars (Hoppe et al. 1997; Zinner et al. 2006). Deep mixing or 'cool bottom processing' during the RG and AGB phases (Wasserburg, Boothroyd \& Sackmann 1995; Langer et al. 1999; Nollett, Busso \& Wasserburg 2003) has been invoked to explain this discrepancy.

Silicon-28 excesses, and especially the original presence of short-lived ${ }^{44} \mathrm{Ti}$ (see below), constitute clear evidence that $\mathrm{SiC}$ grains of type $\mathrm{X}$ come from supernovae. Their generally high ${ }^{12} \mathrm{C} /{ }^{13} \mathrm{C}$ ratios are the signature of $\mathrm{He}$ burning in the $\mathrm{He} / \mathrm{C}$ zone and the observed range of ratios can be explained by mixing with the overlying $\mathrm{He} / \mathrm{N}$ zone where ${ }^{12} \mathrm{C} /{ }^{13} \mathrm{C}$ ratios are low (Meyer, Weaver \& Woosley 1995; Woosley \& Weaver 1995; Rauscher et al. 2002). The ${ }^{15} \mathrm{~N}$ excesses seen in $\mathrm{X}$ grains reflect the high ${ }^{15} \mathrm{~N}$ concentration found in the lower layers of the He/C zone. There ${ }^{15} \mathrm{~N}$ is produced by explosive nucleosynthesis during the passage of the shock front by ${ }^{18} \mathrm{O}(\mathrm{p}, \alpha){ }^{15} \mathrm{~N}$ (see Figure 3), where the protons come from ${ }^{14} \mathrm{~N}(\mathrm{n}, \mathrm{p}){ }^{14} \mathrm{C}$ following ${ }^{22} \mathrm{Ne}(\alpha, n){ }^{25} \mathrm{Mg}$. Another channel for ${ }^{15} \mathrm{~N}$ production is ${ }^{14} \mathrm{~N}(\alpha, \gamma){ }^{18} \mathrm{~F}(\mathrm{p}, \alpha){ }^{15} \mathrm{O}\left(\beta^{+}\right){ }^{15} \mathrm{~N}$ (see Figure 3). Finally, in supernovae ${ }^{15} \mathrm{~N}$ is also produced by absorption of neutrinos from the newly formed neutron stars by ${ }^{16} \mathrm{O}$, ${ }^{16} \mathrm{O}(\nu, \mathrm{n}){ }^{15} \mathrm{O}\left(\beta^{+}\right){ }^{15} \mathrm{~N}$. This is a process that had not been imagined by $\mathrm{B}^{2} \mathrm{FH}$.

The stellar origin of $\mathrm{SiC}$ grains of type $\mathrm{A}+\mathrm{B}$ is still enigmatic. They clearly show the signature of $\mathrm{H}$ burning in the $\mathrm{CNO}$ cycle and $\mathrm{H}$ burning is also indicated by their relatively high inferred ${ }^{26} \mathrm{Al} /{ }^{27} \mathrm{Al}$ ratios (Amari et al. 2001b). However, the low ${ }^{12} \mathrm{C} /{ }^{13} \mathrm{C}$ ratios are difficult to reconcile with the condition $\mathrm{C}>\mathrm{O}$, necessary for $\mathrm{SiC}$ condensation. SiC A $+\mathrm{B}$ grains with low ${ }^{14} \mathrm{~N} /{ }^{15} \mathrm{~N}$ ratios (Figure 3) also pose a problem. J-type carbon stars have low ${ }^{12} \mathrm{C} /{ }^{13} \mathrm{C}$ ratios but their evolutionary history and nucleosynthesis are not well understood. Born-again AGB 


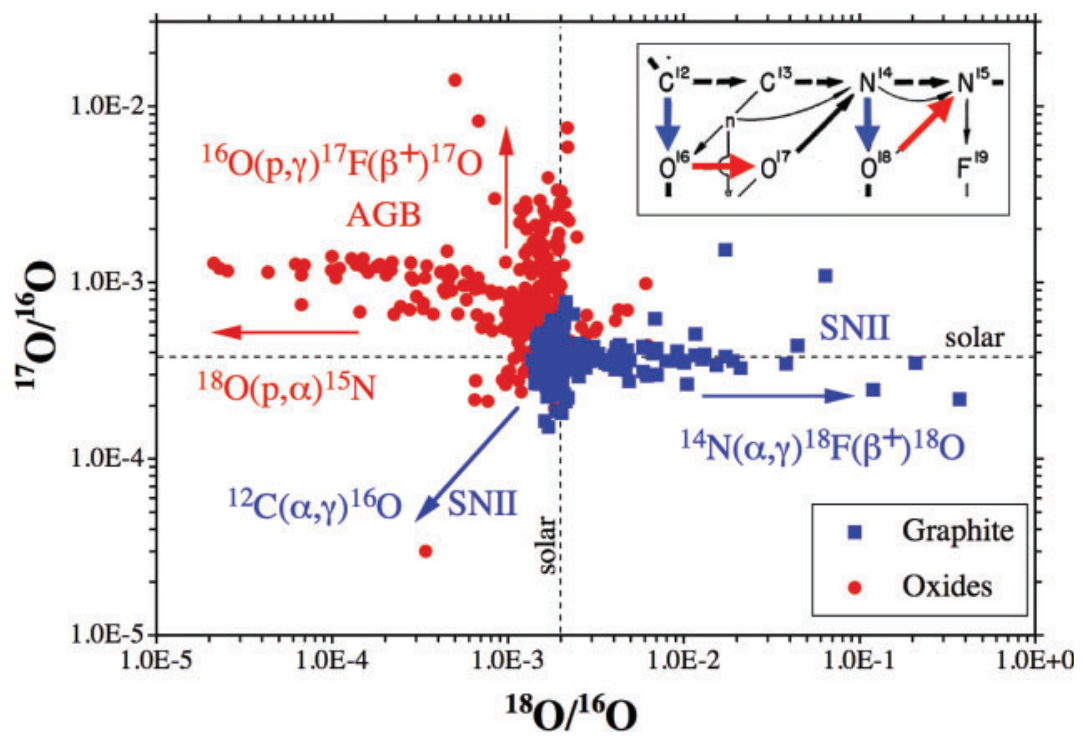

Figure 4 Oxygen isotopic ratios measured in presolar oxide and graphite grains. Proton- and $\alpha$-capture reactions and the inferred stellar sources of the grains are indicated. The insert shows the $\mathrm{p}$ - and $\alpha$-capture reactions from $\mathrm{B}^{2} \mathrm{FH}$.

stars such as Sakurai's object (Asplund et al. 1999) have also been proposed as sources of $\mathrm{A}+\mathrm{B}$ grains (Amari et al. 2001b).

Hydrogen burning in the hot $\mathrm{CNO}$ cycle produces very low ${ }^{12} \mathrm{C} /{ }^{13} \mathrm{C}$ and ${ }^{14} \mathrm{~N} /{ }^{15} \mathrm{~N}$ ratios. This process occurs during nova explosions (José et al. 2004) and a few SiC grains with such a signature (see Figure 3 ) and ${ }^{30} \mathrm{Si}$ excesses are considered to have a nova origin (Amari et al. 2001a).

The signatures of $\mathrm{H}$ and $\mathrm{He}$ burning are also seen in the O isotopic compositions of oxide and low-density graphite grains (Figure 4).

Most oxide grains appear to come from RG and AGB stars. The ${ }^{17} \mathrm{O}$ enrichments in these grains are explained by $\mathrm{H}$ burning in the $\mathrm{CNO}$ cycle in the core of low-tointermediate mass stars during the hydrostatic phase of the parent stars followed by first (and second) dredge-up (Boothroyd, Sackmann \& Wasserburg 1994; Boothroyd \& Sackmann 1999; Nittler et al. 1997). However, the first and second dredge-up produce only modest depletions in ${ }^{18} \mathrm{O}$ and cannot account for the large ${ }^{18} \mathrm{O}$ deficits observed in many grains (Figure 4). Cool bottom processing during the RG and AGB phase has been proposed to explain the compositions of these grains (Wasserburg et al. 1995; Nollett et al. 2003). In this process, material from the bottom of the envelope circulates through hot regions close to the H-burning shell where ${ }^{18} \mathrm{O}$ is effectively destroyed by p capture (Figure 4 ). A single oxide grain shows a large ${ }^{16} \mathrm{O}$ excess, the signature of a Type II supernova, where large amounts of ${ }^{16} \mathrm{O}$ are produced by $\alpha$ capture on ${ }^{12} \mathrm{C}$, which in turn has been made by the triple- $\alpha$ reaction. It remains a puzzle why Type II supernovae, which contain massive amounts of ${ }^{16} \mathrm{O}$ do not produce more such grains.

Many low-density graphite grains contain enough $\mathrm{O}$ for isotopic measurements (Amari, Zinner \& Lewis 1995; Travaglio et al. 1999). They show large ${ }^{18} \mathrm{O}$ excesses relative to solar (Figure 4). These excesses have been interpreted to originate from the He/C shell of Type II supernovae where ${ }^{14} \mathrm{~N}$, left over from the preceding $\mathrm{H}-$ burning phase, is converted to ${ }^{18} \mathrm{O}$ by $\alpha$ capture and subsequent $\beta^{+}$decay (see Figure 4). A SN origin for lowdensity grains is also indicated by other isotopic features such as high inferred ${ }^{26} \mathrm{Al} /{ }^{27} \mathrm{Al}$ ratios (Travaglio et al. 1999), ${ }^{41} \mathrm{Ca} /{ }^{40} \mathrm{Ca}$ ratios (Amari, Zinner \& Lewis 1996) and evidence for ${ }^{44} \mathrm{Ti}$ (Nittler et al. 1996).

Another signature for $\mathrm{H}$ burning displayed by presolar $\mathrm{SiC}$, graphite and oxide grains is evidence for the initial presence of the short-lived $\left(\mathrm{T}_{1 / 2}=735000 \mathrm{yr}\right)$ isotope ${ }^{26} \mathrm{Al}$. This evidence comes in the form of large excesses in ${ }^{26} \mathrm{Mg}$, the daughter of ${ }^{26} \mathrm{Al}$. Aluminum-26 is made by p capture on ${ }^{25} \mathrm{Mg}$. Figure 5 shows a plot of inferred ${ }^{26} \mathrm{Al} /{ }^{27} \mathrm{Al}$ ratios in oxide grains. It has already been mentioned that extra mixing is required to explain ${ }^{18} \mathrm{O} /{ }^{16} \mathrm{O}$ ratios of less than $10^{-3}$.

In AGB stars ${ }^{26} \mathrm{Al}$ is produced in the H-burning shell and brought to the star's surface by third dredge-up. However, shell $\mathrm{H}$ burning can account only for ${ }^{26} \mathrm{Al} /{ }^{27} \mathrm{Al}$ ratios of up to $\sim 3 \times 10^{-3}$ (Forestini, Paulus \& Arnould 1991; Mowlavi \& Meynet 2000; Karakas \& Lattanzio 2003) and cool bottom processing has been invoked for grains with higher ratios (Nollett et al. 2003). This again demonstrates that other processes such as mixing have to be combined with nucleosynthetic reactions in order to explain the isotopic compositions of stellar atmospheres reflected in presolar grains.

\subsection{The $\alpha$-Process}

$\mathrm{B}^{2} \mathrm{FH}$ proposed that the $\alpha$-nuclei such as ${ }^{24} \mathrm{Mg},{ }^{28} \mathrm{Si},{ }^{32} \mathrm{~S}$, up to ${ }^{48} \mathrm{Ti}$ were produced by consecutive $\alpha$-captures on ${ }^{20} \mathrm{Ne}$ (see Figure 2). While, e.g. ${ }^{24} \mathrm{Mg}$ can be produced 


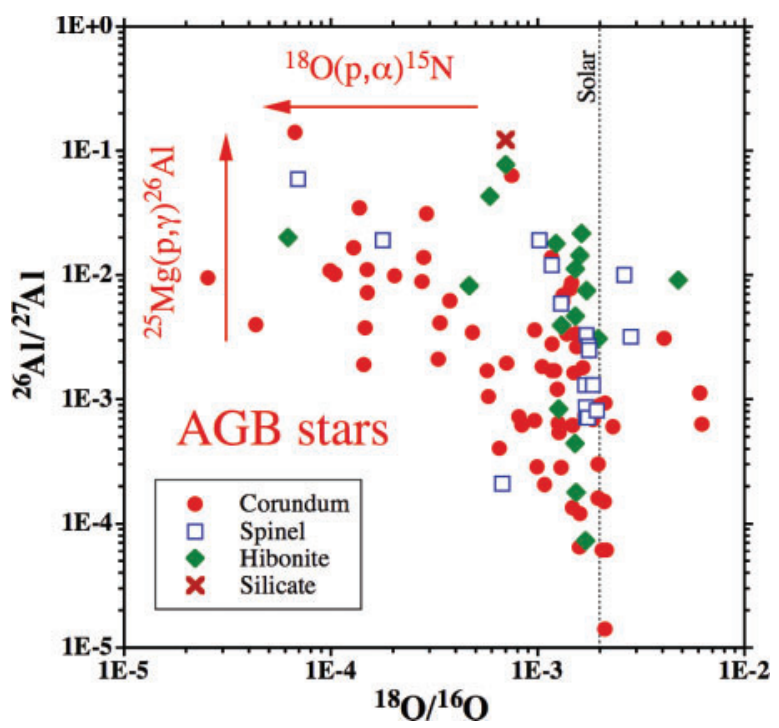

Figure 5 Aluminum isotopic ratios inferred from ${ }^{26} \mathrm{Mg}$ excesses in oxide grains are plotted against their ${ }^{18} \mathrm{O} /{ }^{16} \mathrm{O}$ ratios. The oxide minerals are corundum $=\mathrm{Al}_{2} \mathrm{O}_{3}$, spinel $=\mathrm{Al}_{2} \mathrm{MgO}_{4}$, hibonite $=$ $\mathrm{Al}_{12} \mathrm{CaO}_{19}$. The silicate is amorphous and non-stoichiometric (Nguyen et al. 2007).

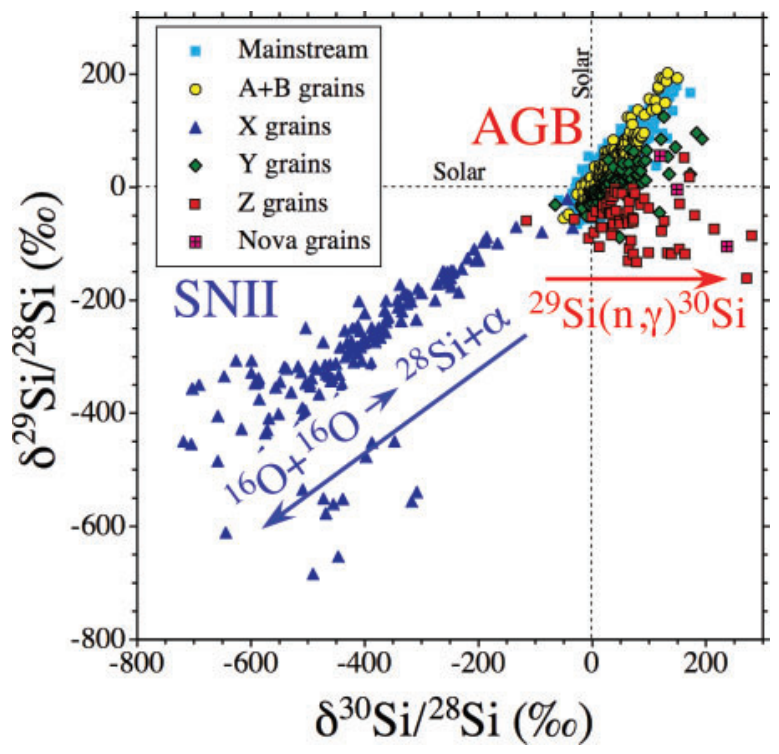

Figure 6 Silicon isotopic ratios of presolar $\mathrm{SiC}$ grains are plotted as $\delta$-values, deviations from the solar ratios in permil $(\%)$. Thus $\delta^{\mathrm{i}} \mathrm{Si}=\left[\left({ }^{\mathrm{i}} \mathrm{Si} /{ }^{28} \mathrm{Si}\right) /\left({ }^{\mathrm{i}} \mathrm{Si} /{ }^{28} \mathrm{Si}\right)_{\text {solar }}-1\right]$, where $\mathrm{i}=29,30$.

by ${ }^{20} \mathrm{Ne}(\alpha, \gamma){ }^{24} \mathrm{Mg}$, today we know that most of these isotopes are produced by reactions between heavier nuclei. For example, ${ }^{12} \mathrm{C}\left({ }^{12} \mathrm{C}, \gamma\right){ }^{24} \mathrm{Mg}$ during so-called carbon burning and ${ }^{16} \mathrm{O}\left({ }^{16} \mathrm{O}, \alpha\right){ }^{28} \mathrm{Si}$ during oxygen burning in massive stars that explode as supernovae. Evidence for $\mathrm{O}$ burning is found in presolar $\mathrm{SiC}$ grains of type $\mathrm{X}$ (Figure 6). In $\mathrm{SN}$ models the $\mathrm{Si} / \mathrm{O}$ and $\mathrm{S} / \mathrm{Si}$ layers below the O-rich zones contain almost pure ${ }^{28} \mathrm{Si}$ (Woosley \& Weaver 1995; Rauscher et al. 2002). To explain the C, N, Al and $\mathrm{Si}$ isotopic ratios and the presence of ${ }^{44} \mathrm{Ti}$ (see below) in $\mathrm{X}$ grains one has to mix material from the inner ${ }^{28} \mathrm{Si}$-rich (and ${ }^{44} \mathrm{Ti}$-containing) zones with material from the outer
$\mathrm{He} / \mathrm{C}$ and He/N zones (Yoshida \& Hashimoto 2004). At the same time, contributions from the massive intermediate O-rich zones have to be severely limited. While astronomical observations give evidence for extensive mixing of SN ejecta (e.g. Hughes et al. 2000), it is an open question whether such mixing can be achieved on a microscopic scale (Deneault, Clayton \& Heger 2003).

Mainstream, $\mathrm{Y}$ and $\mathrm{Z}$ grains, believed to have originated in AGB stars, show some evidence for neutron capture in their $\mathrm{Si}$ isotopic ratios (see below).

\subsection{The Equilibrium Process}

The equilibrium process or $e$-process is responsible for the production of the Fe-peak nuclei. At very high temperatures in the core of massive stars at the end of nuclear burning disintegration reactions break down nuclei such as ${ }^{28} \mathrm{Si}$ (Si-burning) to lighter nucleons and protons and neutrons. These nuclear species are in a nuclear statistical equilibrium and under these conditions nuclei with strong nuclear binding such as $\mathrm{Fe}$ and $\mathrm{Ni}$ are created. At slightly lower temperature a so-called quasi-statistical equilibrium (QSE) is established, in which all nuclei heavier than C are in equilibrium (Meyer, Krishnan \& Clayton 1998). Evidence for the $e$-process is shown by presolar graphite and $\mathrm{SiC}$ grains in the form of the initial presence of the radionuclide ${ }^{44} \mathrm{Ti}\left(\mathrm{T}_{1 / 2}=60 \mathrm{yr}\right.$ ) (Amari et al. 1992; Hoppe et al. 1996; Nittler et al. 1996). Figure 7 shows inferred ${ }^{44} \mathrm{Ti} /{ }^{48} \mathrm{Ti}$ ratios and $\mathrm{Si}$ isotopic ratios (as $\delta$-values) in $\mathrm{SiC}$ grains of type $\mathrm{X}$ and low-density graphite grains. The initial presence of ${ }^{44} \mathrm{Ti}$ is inferred from large excesses in ${ }^{44} \mathrm{Ca}$. The panel on the lower left displays Ca isotopic ratios (normalized to solar ratios) in a graphite grain. Excesses of a factor or 2-3 in ${ }^{42} \mathrm{Ca}$ and ${ }^{43} \mathrm{Ca}$ can be explained by neutron capture. However, neutron capture is predicted to result in a comparable or even smaller excess in ${ }^{44} \mathrm{Ca}$. Instead, ${ }^{44} \mathrm{Ca}$ is in excess by a factor of 140 , leaving little doubt that the decay of ${ }^{44} \mathrm{Ti}$ is responsible for this excess. Because ${ }^{44} \mathrm{Ti}$ is only produced in SN explosions (Timmes et al. 1996), its presence in presolar grains is the most convincing evidence for their SN origin. In Type II supernovae, ${ }^{44} \mathrm{Ti}$ is produced by $\alpha$-rich freeze-out from QSE in the $\mathrm{Si} / \mathrm{S}$ and $\mathrm{Ni}$ zones. Because $\mathrm{Si}$ in the $\mathrm{Si} / \mathrm{S}$ zone is almost pure ${ }^{28} \mathrm{Si}$, it is no surprise that grains with evidence for ${ }^{44} \mathrm{Ti}$ show ${ }^{28} \mathrm{Si}$ excesses (Figure 7).

Another $e$-process signature in presolar grains is found in the form of the initial presence of the radionuclide ${ }^{49} \mathrm{~V}\left(\mathrm{~T}_{1 / 2}=337\right.$ days), indicated by large excesses of the daughter ${ }^{49} \mathrm{Ti}$ (Amari et al. 1992; Nittler et al. 1996; Travaglio et al. 1999; Hoppe \& Besmehn 2002). While ${ }^{49} \mathrm{Ti}$ excesses can also be produced by neutron capture in the He/C zone, Hoppe and Besmehn (2002) claimed, on the basis of a correlation between ${ }^{49} \mathrm{Ti}$ excesses and the V/Ti ratio in $\mathrm{X}$ grains, that ${ }^{49} \mathrm{~V}$ decay was responsible. Travaglio et al. (1999) found that the ${ }^{49} \mathrm{Ti}$ excesses in low-density graphite grains with ${ }^{12} \mathrm{C} /{ }^{13} \mathrm{C}<100$ can only be explained by mixing of SN layers if ${ }^{49} \mathrm{~V}$ decay is considered. Like ${ }^{44} \mathrm{Ti},{ }^{49} \mathrm{~V}$ is produced by $\alpha$-rich freeze-out in the $\mathrm{Si} / \mathrm{S}$ and $\mathrm{Ni}$ zones. 

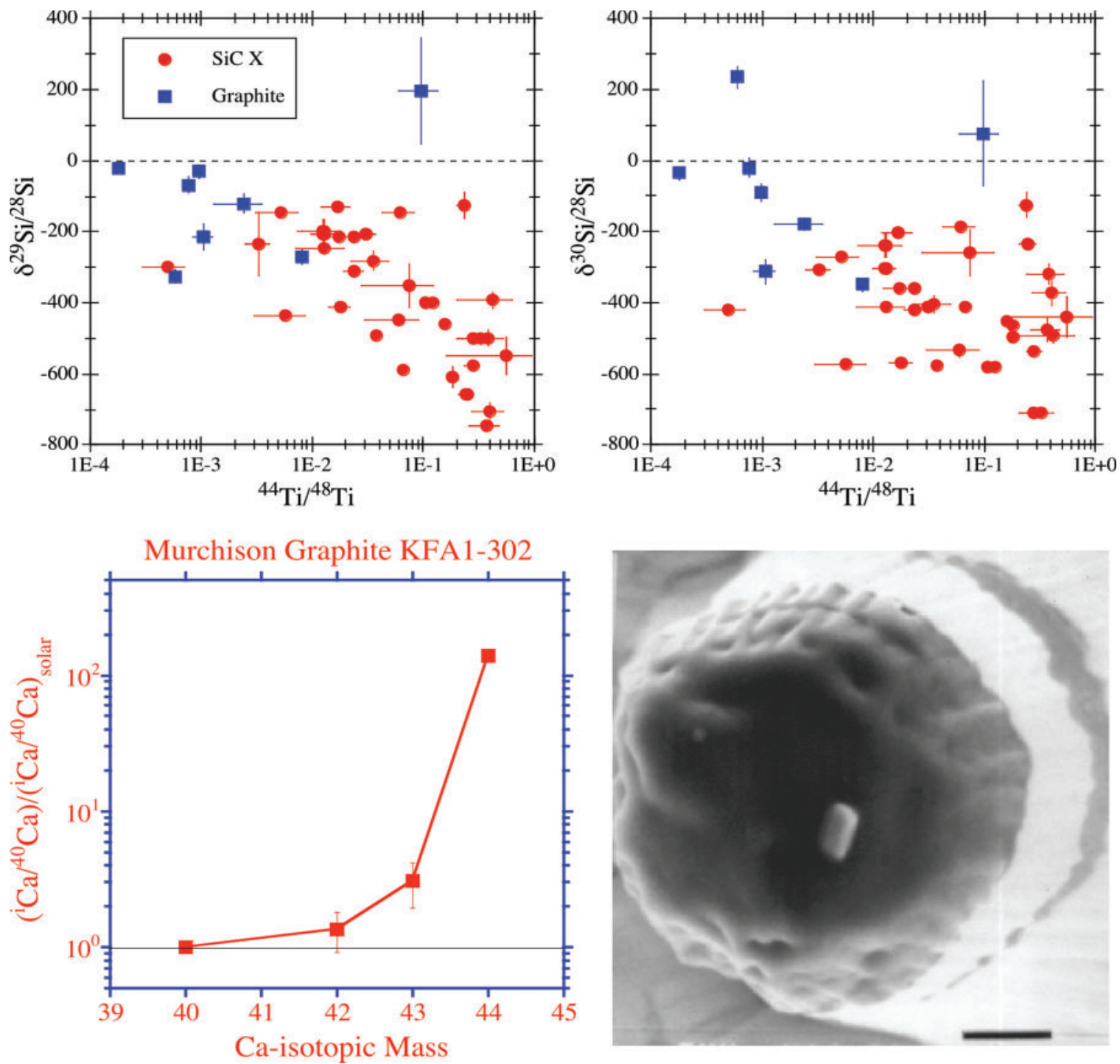

Figure 7 Upper two panels: Si isotopic ratios of $\mathrm{SiC} \mathrm{X}$ and graphite grains are plotted against inferred ${ }^{44} \mathrm{Ti} /{ }^{48} \mathrm{Ti}$ ratios. Lower-left panel: $\mathrm{Ca}$ isotopic ratios of a graphite grain KFA1-302 from the Murchison meteorite. Lower-right panel: secondary electron micrograph of grain KFA1-302. The image shows Ti carbide sub-grain, which apparently carried the original ${ }^{44} \mathrm{Ti}$ whose decay led to a large ${ }^{44} \mathrm{Ca}$ excess.

\subsection{The s-Process}

The $s$-process is neutron capture at neutron densities that are low enough that radionuclides can $\beta$-decay back to the valley of stability before they capture another neutron. The isotopic ratios of the heavy elements in $\mathrm{SiC}$ grains show the signature of the $s$-process (Figure 8). This signature is a characteristic zig-zag patterns in the isotopic abundances between isotopes with even and odd numbers of nucleons, reflecting their neutron-capture cross sections. Because mainstream grains dominate among presolar $\mathrm{SiC}$ grains, the $s$-process signature must be carried by these grains. All patterns shown in Figure 8 can be successfully reproduced by models of low-mass AGB stars (Gallino et al. 1997b; Arlandini et al. 1999; Lugaro et al. 2003, 2004). Discrepancies with earlier model calculations were caused by incorrect n-capture cross sections and could be resolved by improved cross-section measurements (Guber et al. 1997; Wisshak et al. 1997; Koehler et al. 1998). In AGB stars, believed to be the source of the main component of the $s$-process, neutrons are produced by $\mathrm{He}$ burning in the He intershell. Two neutron sources have been identified: ${ }^{13} \mathrm{C}(\alpha, \mathrm{n}){ }^{16} \mathrm{O}$ and ${ }^{22} \mathrm{Ne}(\alpha, \mathrm{n}){ }^{25} \mathrm{Mg}$. Neutrons from the first source are produced in the radiative intershell between thermal pulses, those from the second source during the short thermal pulse when the intershell is convective (Gallino et al. 1998; Busso, Gallino \& Wasserburg 1999). The heavy elements are mostly affected by the ${ }^{13} \mathrm{C}$ source.

In models of AGB nucleosynthesis a ${ }^{~}{ }^{13} \mathrm{C}$ pocket' is assumed to form by ingestions of protons from the $\mathrm{H}$-rich envelope into the ${ }^{12} \mathrm{C}$-rich intershell but this process is not well understood and the strength of the ${ }^{13} \mathrm{C}$ pocket is 

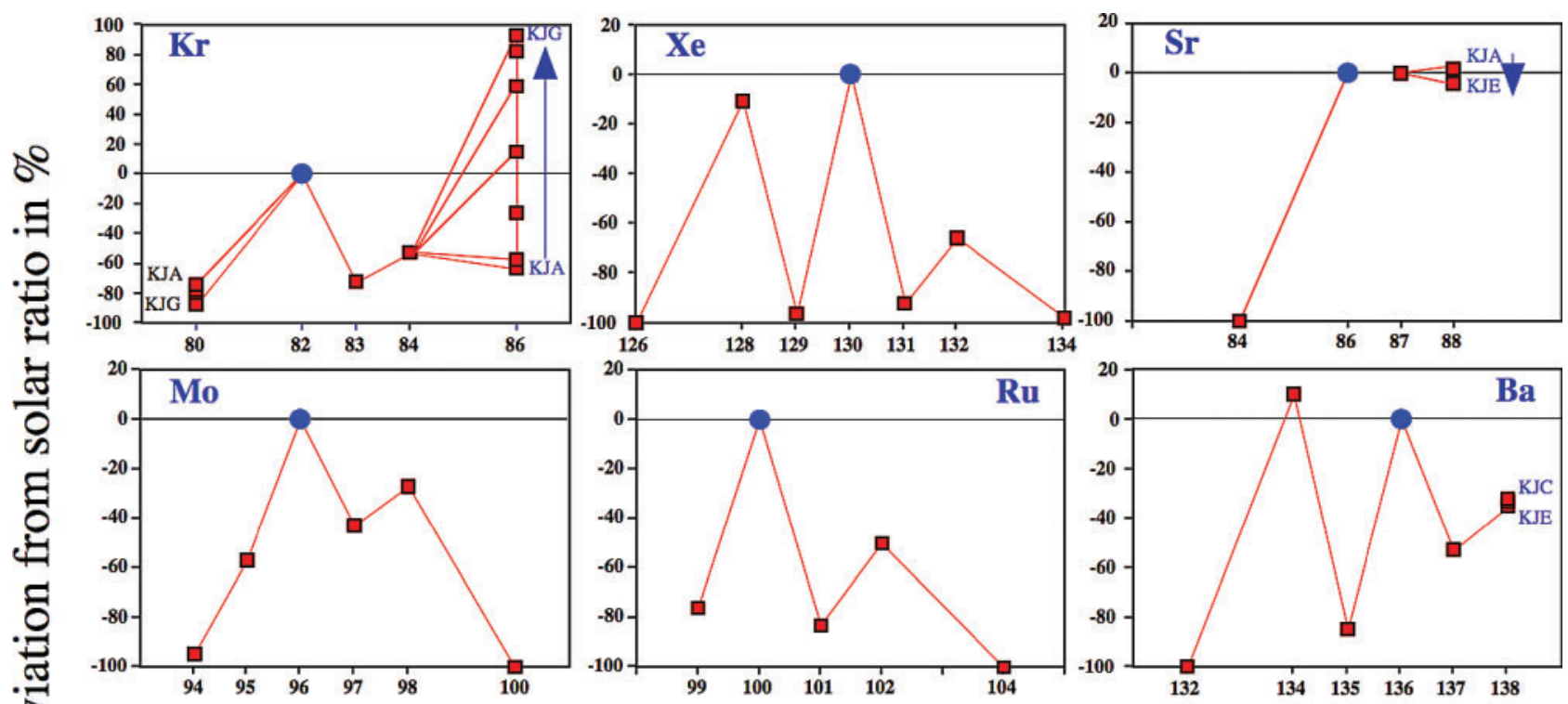

คั
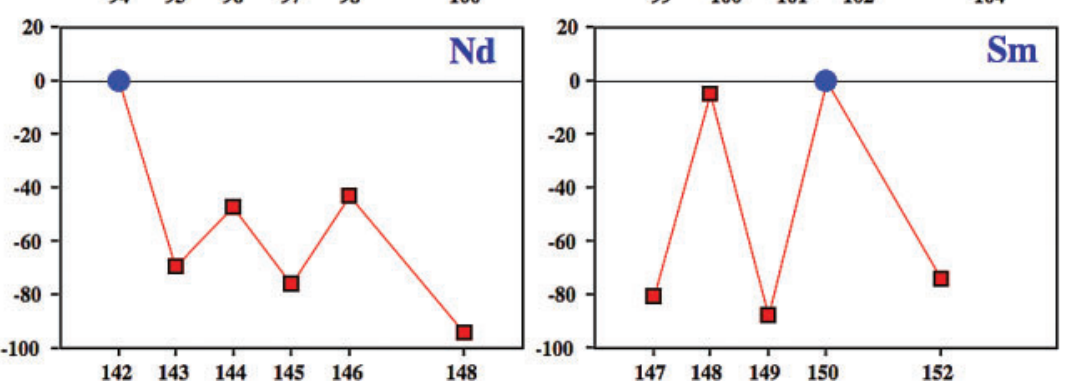

\section{s-process}

\section{AGB stars}

Figure 8 Isotopic patterns of the heavy elements in presolar $\mathrm{SiC}$ grains. Isotopic patterns are relative to the reference isotope plotted as a solid circle and are normalized to the Solar isotopic ratios. Plotted are the pure $s$-process ratios that exist in the He shell of AGB stars.

essentially a free parameter. Recent RIMS and NanoSIMS measurements on mainstream $\mathrm{SiC}$ grains indicate a limited range in the strength of the ${ }^{13} \mathrm{C}$ pocket (Barzyk et al. 2007; Marhas, Hoppe \& Ott 2007).

The $s$-process is also seen in the $\mathrm{Si}$ and $\mathrm{Ti}$ isotopic ratios of presolar $\mathrm{SiC}$ grains. In Figure 6, Si isotopic ratios of $\mathrm{Z}$ grains, believed to come from low-metallicity AGB stars, plot to the right of the correlation line defined by the mainstream grains. The isotopic shift of the $\mathrm{Si}$ isotopic ratios of these grains, mainly in the ${ }^{30} \mathrm{Si} /{ }^{28} \mathrm{Si}$ ratio, is interpreted to be caused by neutron capture (Lugaro et al. 1999; Zinner et al. 2006). Similarly, isotopic shifts in the $\mathrm{Ti}$ isotopic ratios, mainly in ${ }^{50} \mathrm{Ti} /{ }^{48} \mathrm{Ti}$, in type- $\mathrm{Z} \mathrm{SiC}$ grains are apparently the result of neutron capture (Zinner et al. 2007).

A triumph of sorts was achieved with the finding of evidence for the initial presence of ${ }^{99} \mathrm{Tc}$ in presolar $\mathrm{SiC}$ grains (Figure 9). Remember that the discovery of Tc in a star (Merrill 1952) was proof of stellar nucleosynthesis and the radioisotope ${ }^{99} \mathrm{Tc}\left(t_{1 / 2}=0.213 \mathrm{Myr}\right)$ was specifically listed by $\mathrm{B}^{2} \mathrm{FH}$ as an $s$-process product (see Figure 2). The $\mathrm{Ru}$ isotopic ratios agree with predictions from AGB models only if the decay of ${ }^{99} \mathrm{Tc}$ is included. This was interpreted as evidence for the presence of ${ }^{99} \mathrm{Tc}$ at the time of grain formation (Savina et al. 2004).

\subsection{The r-Process}

With the possible exception of the heavy Xe isotopes in nanodiamonds (Lewis et al. 1987), no convincing evidence for the $r$-process, the addition of neutrons at very high neutron densities, has been found in stardust grains. SiC grains of type X show an isotopic pattern (Figure 10) that does not show any evidence for the $r$-process, such as a large ${ }^{100}$ Mo excess, but that can be explained by a short intense neutron burst (Meyer, Clayton \& The 2000). Recent models of Type II supernovae (Rauscher et al. 2002) show that a neutron burst occurs in a thin layer just below the $\mathrm{He} / \mathrm{C}$ zone that can account for the Mo isotopic data. In this shell large amounts of the radioisotope ${ }^{60} \mathrm{Fe}\left(t_{1 / 2}=1.5 \mathrm{Myr}\right)$ are also produced but searches for evidence of ${ }^{60} \mathrm{Fe}$ in presolar grains have been negative to date.

\subsection{The p-Process}

The $p$-process has been postulated by $\mathrm{B}^{2} \mathrm{FH}$ to explain the existence of several p-rich heavy nuclei. Today it is believed that the process responsible for the production of these nuclei is photodisintegration (Woosley \& Howard 1978). To date only one example of the $p$-process signature in presolar grains has been found in the form of excesses of the $p$-process isotopes ${ }^{92,94} \mathrm{Mo}$ and ${ }^{96,98} \mathrm{Ru}$ in a $\mathrm{SiC}$ grain 


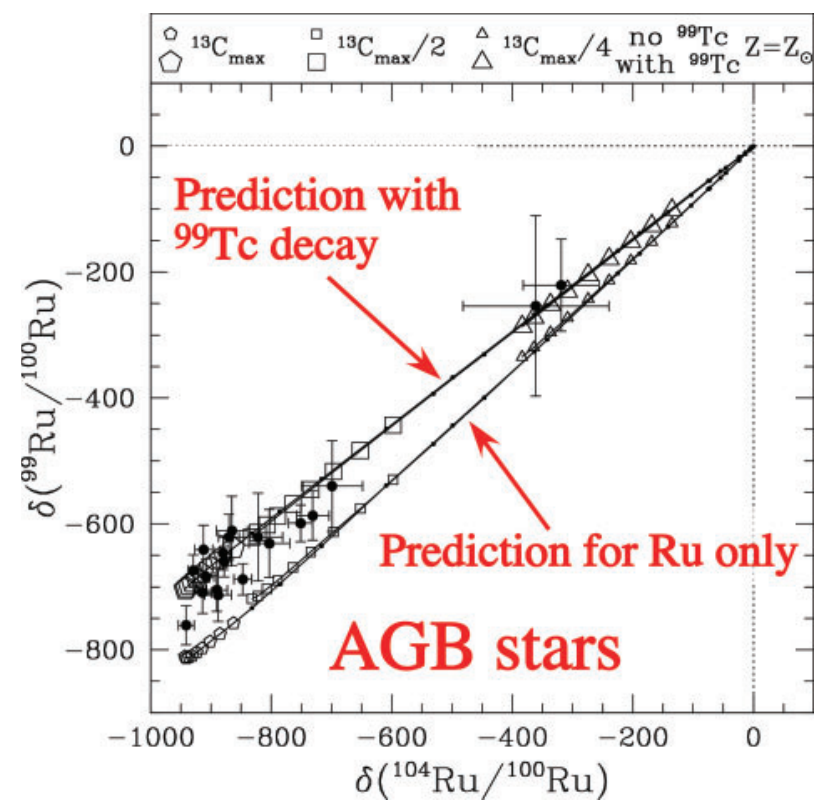

Figure 9 Ruthenium isotopic ratios measured by RIMS in mainstream $\mathrm{SiC}$ grains. The solid symbols are RIMS data, the open symbols theoretical predictions for ratios in the envelope of AGB stars of Solar metallicity. The data agree with the models only if in situ decay of ${ }^{99} \mathrm{Tc}$ is included. The figure is from Lugaro (2005).

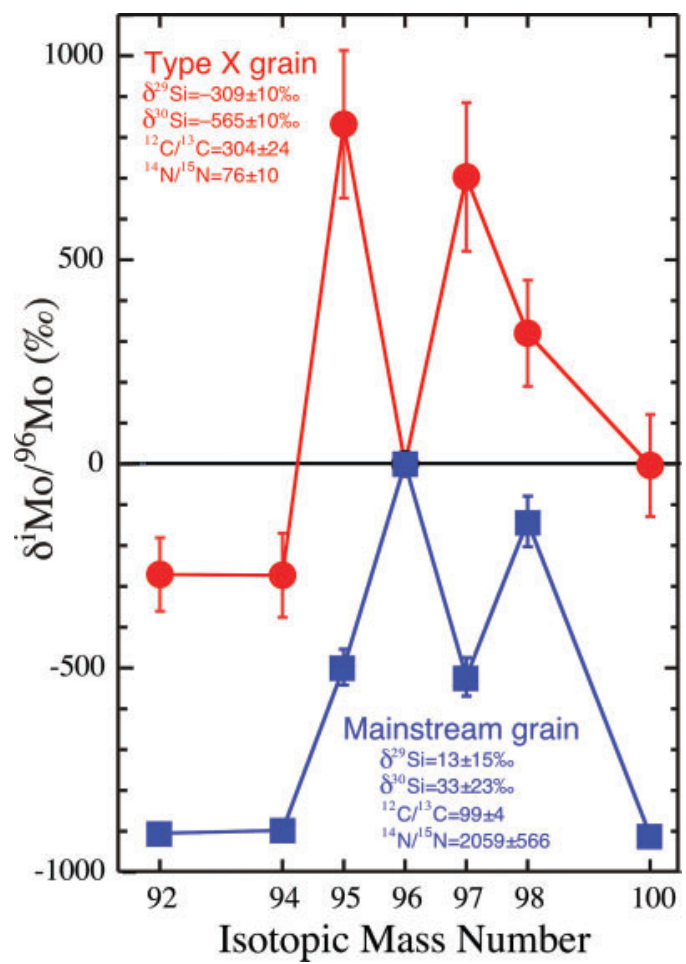

Figure 10 Molybdenum isotopic pattern measured by RIMS in a type $\mathrm{X}$ and a mainstream $\mathrm{SiC}$ grain. The figure is from Pellin et al. (1999).

(Figure 11). The RIMS measurements shown in the figure were obtained on a $\mathrm{SiC}$ grain with ${ }^{12} \mathrm{C} /{ }^{13} \mathrm{C}=4.5$. While mixing of the inner O-rich zone of Type II supernovae (Rauscher et al. 2002) with isotopic normal material
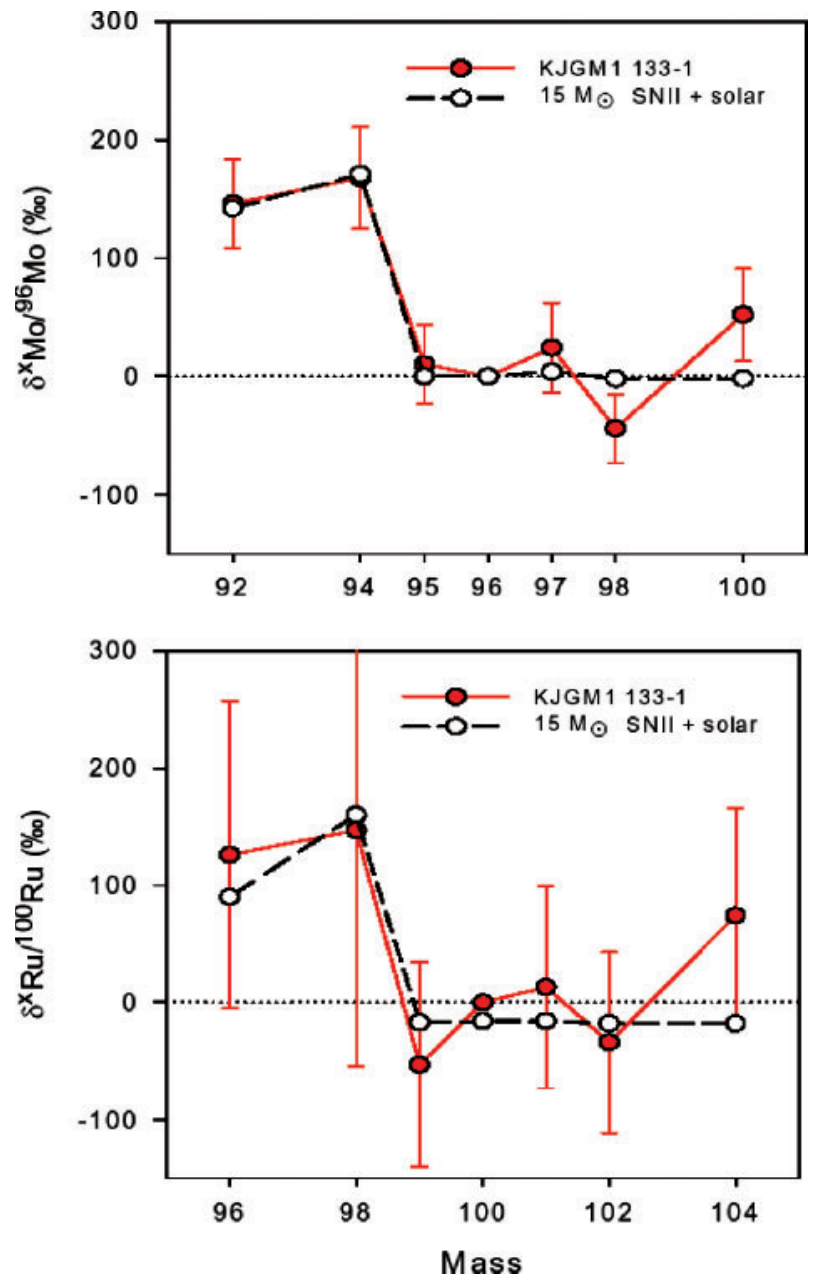

Figure 11 Molybdenum and $\mathrm{Ru}$ isotopic patterns measured in a type $\mathrm{A}+\mathrm{B} \mathrm{SiC}$ grain. The open symbols are predictions for a mix from the interior of a $15 \mathrm{M}_{\odot}$ supernova and material of solar composition. The figure is from Savina et al. (2007).

can produce the $p$-process patterns in Mo and $\mathrm{Ru}$ (Figure 11), the $\mathrm{C}, \mathrm{N}$ and $\mathrm{Si}$ isotopic compositions of the grain cannot be reproduced in this way (Savina et al. 2007).

\subsection{The x-Process}

The $x$-process is responsible for the production of the light elements $\mathrm{Li}, \mathrm{Be}$ and $\mathrm{B}$. These elements are made by spallation reactions on heavier elements such as $\mathrm{C}$. While $\mathrm{B}^{2} \mathrm{FH}$ considered these reactions to take place in stellar atmospheres, we now know that the light elements are produced in the interstellar medium by Galactic cosmic rays. There might be some evidence for this process in stardust grains. Gyngard et al. (2007) observed excesses in ${ }^{6} \mathrm{Li}$ and ${ }^{10} \mathrm{~B}$ relative to the Solar abundances in presolar $\mathrm{SiC}$ grains. They interpreted these excesses to be produced by spallation reactions on the $\mathrm{C}$ in the grains. If this is the case, such excesses can be used to determine the residence time of presolar $\mathrm{SiC}$ grains in the interstellar medium.

\section{Conclusions}

The study of stardust in the laboratory has already provided a wealth of isotopic data in different types of grains. 
Of special interests are results that do not agree with existing models of stellar nucleosynthesis and thus lead to the introduction of new models (e.g. cool bottom processing), the measurements of cross sections $(\mathrm{Ba}, \mathrm{Nd})$, or the search of new processes not considered before (neutron burst). Recent instrumental advances (e.g. the NanoSIMS) have resulted in the analysis of smaller and smaller grains. More RIMS data will undoubtedly lead to new insights into stellar nucleosynthesis.

\section{Acknowledgments}

I thank John Lattanzio for encouraging me to write this article. Sachiko Amari, Roberto Gallino, Frank Gyngard, Peter Hope, Manavi Jadhav, Maria Lugaro, Larry Nittler and Mike Savina have contributed in one way or another to this paper. Almost all the grain data presented in the figures can be found in the Presolar Database at http://presolar.wustl.edu/ pgd/. This work was supported by NASA grant NNG05GF81G.

\section{References}

Amari, S., Anders, E., Virag, A. \& Zinner, E., 1990, Natur, 345, 238 Amari, S., Hoppe, P., Zinner, E. \& Lewis, R. S., 1992, ApJ, 394, L43 Amari, S., Lewis, R. S. \& Anders, E., 1994, GeCoA, 58, 459

Amari, S., Lewis, R. S. \& Anders, E., 1995, GeCoA, 59, 1411

Amari, S., Zinner, E. \& Lewis, R. S., 1995, ApJ, 447, L147

Amari, S., Zinner, E. \& Lewis, R. S., 1996, ApJ, 470, L101

Amari, S., Gao, X., Nittler, L. R., Zinner, E., José, J., Hernanz, M. \& Lewis, R. S., 2001a, ApJ, 551, 1065

Amari, S., Nittler, L. R., Zinner, E., Lodders, K. \& Lewis, R. S., 2001b, ApJ, 559, 463

Anders, E. \& Grevesse, N., 1989, GeCoA, 53, 197

Anders, E. \& Zinner, E., 1993, Metic, 28, 490

Arlandini, C., Käppeler, F., Wisshak, K., Gallino, R., Lugaro, M., Busso, M. \& Straniero, O., 1999, ApJ, 525, 886

Asplund, M., Grevesse, N. \& Sauval, A. J., 2005, in ASPC 336, Cosmic Abundances as Records of Stellar Evolution and Nucleosynthesis, Eds. Barnes, T. G. \& Bash, F. N. (San Francisco: ASP), 25

Asplund, M., Lambert, D. L., Kipper, T., Pollacco, D. \& Shetrone, M. D., 1999, A\&A, 343, 507

Barzyk, J. G. et al., 2007, M\&PS, 42, 1103

Bernatowicz, T., Fraundorf, G., Tang, M., Anders, E., Wopenka, B., Zinner, E. \& Fraundorf, P., 1987, Natur, 330, 728

Bernatowicz, T. J., Cowsik, R., Gibbons, P. C., Lodders, K., Fegley, B. Jr, Amari, S. \& Lewis, R. S., 1996, ApJ, 472, 760

Boothroyd, A. I. \& Sackmann, I.-J., 1999, ApJ, 510, 232

Boothroyd, A. I., Sackmann, I.-J. \& Wasserburg, G. J., 1994, ApJ, 430, L77

Burbidge, E. M., Burbidge, G. R., Fowler, W. A. \& Hoyle, F., 1957, RvMP, 29, 547

Busso, M., Gallino, R. \& Wasserburg, G. J., 1999, ARA\&A, 37, 239

Cameron, A. G. W., 1957, PASP, 69, 201

Cameron, A. G. W., 1973, SSRv, 15, 121

Clayton, D. D. \& Nittler, L. R., 2004, ARA\&A, 42, 39

Croat, T. K., Bernatowicz, T., Amari, S., Messenger, S. \& Stadermann, F. J., 2003, GeCoA, 67, 4705

Deneault, E. A.-N., Clayton, D. D. \& Heger, A., 2003, ApJ, 594, 312

El Eid, M., 1994, A\&A, 285, 915

Forestini, M., Paulus, G. \& Arnould, M., 1991, A\&A, 252, 597

Gallino, R., Raiteri, C. M., Busso, M. \& Matteucci, F., 1994, ApJ, 430,858

Gallino, R., Arlandini, C., Lugaro, M., Busso, M. \& Straniero, O., 1997a, NuPhA, 621, 423c
Gallino, R., Busso, M. \& Lugaro, M., 1997b, in Astrophysical Implications of the Laboratory Study of Presolar Materials, Eds. Bernatowicz, T. J. \& Zinner, E. (New York: AIP), 115

Gallino, R., Arlandini, C., Busso, M., Lugaro, M., Travaglio, C., Straniero, O., Chieffi, A. \& Limongi, M., 1998, ApJ, 497, 388

Grevesse, N., Noels, A. \& Sauval, A. J., 1996, in Cosmic Abundances, Eds. Holt, S. S. \& Sonneborn, G. (San Francisco: BookCrafters, Inc.), 117

Guber, K. H., Spencer, R. R., Koehler, P. E. \& Winters, R. R., 1997, PhRvL, 78, 2704

Gyngard, F., Amari, S., Zinner, E., Gallino, R. \& Lewis, R. S., 2007, in LPSC XXXVIII, 1963

Hoppe, P., 2004, in Astrophysics of Dust, Eds. Witt, A., Clayton, G. C. \& Draine, B. T. (ASP Conf Series 309), 265

Hoppe, P. \& Ott, U., 1997, in Astrophysical Implications of the Laboratory Study of Presolar Materials, Eds. Bernatowicz, T. J. \& Zinner, E. (New York: AIP), 27

Hoppe, P. \& Zinner, E. 2000, JGR, 105, 10371

Hoppe, P. \& Besmehn, A., 2002, ApJ, 576, L69

Hoppe, P., Strebel, R., Eberhardt, P., Amari, S. \& Lewis, R. S., 1996, Sci, 272, 1314

Hoppe, P., Annen, P., Strebel, R., Eberhardt, P., Gallino, R., Lugaro, M., Amari, S. \& Lewis, R. S., 1997, ApJ, 487, L101

Hoyle, F., 1946, MNRAS, 106, 343

Hughes, J. P., Rakowski, C. E., Burrows, D. N. \& Slane, P. O., 2000, ApJ, 528, L109

Hutcheon, I. D., Huss, G. R., Fahey, A. J. \& Wasserburg, G. J., 1994, ApJ, 425, L97

José, J., Hernanz, M., Amari, S., Lodders, K. \& Zinner, E., 2004, ApJ, 612, 414

Käppeler, F., Beer, H. \& Wisshak, K., 1989, RPPh, 52, 945

Karakas, A. I. \& Lattanzio, J. C., 2003, PASA, 20, 279

Koehler, P. E. et al., 1998, PhRvC, 57, R1558

Lambert, D. L., 1991, in Evolution of Stars: The Photospheric Abundance Connection, Eds. Michaud, G. \& Tutukov, A. (Dordrecht: Kluwer), 451

Langer, N., Heger, A., Wellstein, S. \& Herwig, F., 1999, A\&A, 346, L37

Lewis, R. S., Amari, S. \& Anders, E., 1994, GeCoA, 58, 471

Lewis, R. S., Tang, M., Wacker, J. F., Anders, E. \& Steel, E., 1987, Natur, 326, 160

Lodders, K., 2003, ApJ, 591, 1220

Lodders, K. \& Amari, S., 2005, ChEG, 65, 93

Lugaro, M., 2005, Stardust from Meteorites (Singapore: World Scientific), 9, 209

Lugaro, M., Zinner, E., Gallino, R. \& Amari, S., 1999, ApJ, 527, 369

Lugaro, M., Davis, A. M., Gallino, R., Pellin, M. J., Straniero, O. \& Käppeler, F., 2003, ApJ, 593, 486

Lugaro, M., Davis, A. M., Gallino, R., Savina, M. R. \& Pellin, M. J., 2004, MmSAI, 75, 723

Marhas, K. K., Hoppe, P. \& Ott, U., 2007, M\&PS, 42, 1077

Merrill, P. W., 1952, ApJ, 116, 21

Messenger, S., Keller, L. P., Stadermann, F. J., Walker, R. M. \& Zinner, E., 2003, Sci, 300, 105

Meyer, B. S., Weaver, T. A. \& Woosley, S. E., 1995, Metic, 30, 325

Meyer, B. S., Krishnan, T. D. \& Clayton, D. D., 1998, ApJ, 498, 808

Meyer, B. S., Clayton, D. D. \& The, L.-S., 2000, ApJ, 540, L49

Mowlavi, N. \& Meynet, G., 2000, A\&A, 361, 959

Nagashima, K., Krot, A. N. \& Yurimoto, H., 2004, Natur, 428, 921

Nguyen, A. N. \& Zinner, E., 2004, Sci, 303, 1496

Nguyen, A., Zinner, E. \& Lewis, R. S., 2003, PASA, 20, 382

Nguyen, A. N., Stadermann, F. J., Zinner, E., Stroud, R. M., Alexander, C. M. O’D. \& Nittler, L. R., 2007, ApJ, 656, 1223

Nittler, L. R., 2003, E\&PSL, 209, 259

Nittler, L. R., Alexander, C. M. O’D., Gao, X., Walker, R. M. \& Zinner, E. K., 1994, Natur, 370, 443

Nittler, L. R. et al., 1995, ApJ, 453, L25

Nittler, L. R., Amari, S., Zinner, E., Woosley, S. E. \& Lewis, R. S., 1996, ApJ, 462, L31 
Nittler, L. R., Alexander, C. M. O’D., Gao, X., Walker, R. M. \& Zinner, E., 1997, ApJ, 483, 475

Nollett, K. M., Busso, M. \& Wasserburg, G. J., 2003, ApJ, 582, 1036

Pellin, M. J., Davis, A. M., Lewis, R. S., Amari, S. \& Clayton, R. N., 1999, LPSC XXX, 1969

Rauscher, T., Heger, A., Hoffman, R. D. \& Woosley, S. E., 2002, ApJ, 576, 323

Russell, S. S., Arden, J. W. \& Pillinger, C. T., 1996, M\&PS, 31, 343

Russell, S. S., Ott, U., Alexander, C. M. O’D., Zinner, E. K., Arden, J. W. \& Pillinger, C. T., 1997, M\&PS, 32, 719

Savina, M. R., Pellin, M. J., Tripa, C. E., Veryovkin, I. V., Calaway, W. F. \& Davis, A. M., 2003, GeCoA, 67, 3215

Savina, M. R., Davis, A. M., Tripa, C. E., Pellin, M. J., Gallino, R., Lewis, R. S. \& Amari, S., 2004, Sci, 303, 649

Savina, M. R., Pellin, M. J., Davis, A. M., Lewis, R. S., \& Amari, S. 2007, LPSC XXXVIII, 2231

Suess, H. E. \& Urey, H. C., 1956, RvMP, 28, 53

Tang, M. \& Anders, E., 1988, GeCoA, 52, 1235

Timmes, F. X., Woosley, S. E. \& Weaver, T. A., 1995, ApJS, 98, 617

Timmes, F. X., Woosley, S. E., Hartmann, D. H. \& Hoffman, R. D., 1996, ApJ, 464, 332
Travaglio, C., Gallino, R., Amari, S., Zinner, E., Woosley, S. \& Lewis, R. S., 1999, ApJ, 510, 325

Wallerstein, G. et al., 1997, RvMP, 69, 995

Wasserburg, G. J., Boothroyd, A. I. \& Sackmann, I.-J., 1995, ApJ, 447, L37

Wisshak, K., Voss, F., Käppeler, F. \& Kazakov, L., 1997, NuPhA, $621,270 \mathrm{c}$

Woosley, S. E. \& Howard, W. M., 1978, ApJS, 36, 285

Woosley, S. E. \& Weaver, T. A., 1995, ApJS, 101, 181

Yin, Q.-A., Lee, C.-T. \& Ott, U., 2006, ApJ, 647, 676

Yong, D., Lambert, D. L. \& Ivans, I. I., 2003, ApJ, 599, 1357

Yoshida, T. \& Hashimoto, M., 2004, ApJ, 606, 592

Yurimoto, H., Nagashima, K. \& Kunihiro, T., 2003, ApSS, 203-204 793

Zinner, E., 1998, AREPS, 26, 147

Zinner, E., 2007, in Treatise on Geochemistry Update, Eds Holland, H. D., Turekian, K. K. \& Davis, A. (Oxford: Elsevier), 1.02 (online update only), 1

Zinner, E., Nittler, L. R., Gallino, R., Karakas, A. I., Lugaro, M., Straniero, O. \& Lattanzio, J. C., 2006, ApJ, 650, 350

Zinner, E. et al., 2007, GeCoA, 71, 4786 